\title{
List of reviewers 2021
}

On behalf of the editorial board, the Editor-in-Chief would like to thank the following for their contribution as peer reviewers in the period from January 2021 - December 2021. Their anonymous work for the journal forms the foundation to its success, and is highly appreciated.

Melanie Abas

Mohammed Abousaleh

Balkozar Adams

Leslie Adams

Armita Adily

Vivek Agarwal

Bruno Agustini

Suhana Ahmed

Olesya Ajnakina

Hasanen Al-Taiar

Faruq Alam

Regi Alexander

Afia Ali

Abdulmajeed Alkhamees

Norah Almuhanna

Daniel Alvarez-Fischer

Flavia Alves

Shereen Aly

Ahmad Alzahrani

Humma Andleeb

Andrea de Angelis

Afifa Anjum

Paul Appelbaum

S.M. Yasir Arafat

Luis Araujo

Dave Archard

Angeliki Argyriou

Kevin Ariyo

Rehan Aziz

Enrique Baca-Garcia

Ajay Bakhla

Ioannis Bakolis

Azza Bakry

David Baldwin

Matteo Balestrieri

Zoe Barczyk

Kirsten Barnicot

Lucie Bartova

Markus Bassler

Joy Noel Baumgartner

Anna Baverstock

Klaus Martin Beckmann

Victoria Bein

Victoria Bell

Matthew Bennion

Tomi Bergström

Luca Bernardi

Tom Berney

Wade Berrettini

Marco Bertelli

Frank Besag

Venkat Bhat

Vishal Bhavsar

Kamaldeep Bhui

Andrew Bickle

Eleonora Bielawska-Batorowicz

Rebecca Bind

Victoria Bird

Catherine Bird
Mengesha Biresaw

Jonathan Bisson

Asit Biswas

Istvan Bitter

Donald Black

Rebecca Blackmore

Jed Boardman

Harm Boer

Lana Bojanić

Rohan Borschmann

Hans Bosma

Michel Botbol

Theo Bouman

Nick Bouras

Juliette Bouzy

Phillip Boyce

Anders Boyd

Tim Bradshaw

Bethany Brand

Erica Breuer

Sofia Brissos

Andrew Brittlebank

Jillian Broadbear

Carlinde Broeks

Emma Broglia

Konstantin Brückmann

Xuan $\mathrm{Bu}$

Paola Bucci

Dejan Budimirovic

Oscar Bukstein

Katherine Burdick

Julian Burger

Rochelle Burgess

Wendy Burn

Walter Busuttil

Katherine Button

Niels Buus

Amanda Bye

Eric Caine

Elizabeth Camacho

Jose Antonio Camacho Conde

Andres Camilo Cardozo Alarcon

Sara Carletto

Marina Carpena

Peter Carpenter

Holly Carter

Janet Carter

Gustavo Carvalho de Oliveira

Cecilia Casetta

Patricia Casey

Rakesh Chadda

Samuel Chamberlain

Carlyle Chan

Tarani Chandola

Prabha Chandra

Imran Chaudhry

Qingwei Chen

Runsen Chen

Gang Chen
Andrew Cheng
Helen Cheyne
Rie Chiba
Jennifer Chipps
Lois Choi-Kain
Ciaran S. Clarke
Seonaid Cleare
Catherine Clelland
Caroline Clements

Marylene Cloitre

John Cookson

Zafra Cooper

Christine Cooper Vince

Peter Cornwall

Ana Costa

Sara Costi

Ken Courtenay

Lesley Cousins

Tom Craig

John Craig

Mike Crawford

Matthew Croucher

Marie Crowe

Wiesław Cubała

Ruth Cunningham

Qin Dai

Elias Dakwar

Oliver Dale

Christian Dalton-Locke

Michael Daly

Anna-Karin Danielsson

Jayati Das-Munshi

Oana David

Thandi Davies

Katrina Davis

Enya Daynes

Johannes De Kock

Wendy Dean

Kimberlie Dean

Ian Deary

Ana Teresa Delfino

Kutay Demirkan

Mekdes Demissie

Brecht Devleesschauwer

Annabella Digiorgio

Gina Dimitropoulos

Lora Dimitrova

Jade Donaghy

Martin Dorahy

Katie Douglas

Christopher Dowrick

Małgorzata Dragan

Bernadka Dubicka

Coralie Dumoulin

Julian Eaton

Kate Eggleston

Sharif El-Leithy

Angelika Erhardt-Lehmann

Javier Escobar 
Bruno Etain

Kerri Evans

Anne Eyre

Hanan Ez elarab

Nichole Fairbrother

Hanna Falk Erhag

Chao Fang

Miguel Farias

Lorna Farquharson

Daniel Fatori

Seena Fazel

Abe Fekadu

Johannes Fellinger

Peter Fenwick

Lucrezia Ferrante

Laura Ferraro

Emily Finch

Mudasir Firdosi

Claudia Fischer

Emla Fitzsimons

Lena Flyckt

Marcella Fok

Luciana Fonseca

Leonardo Fontenelle

Cheryl Foo

Malcom Forbes

Tamsin Ford

David Foreman

Andrew Forrester

Sarah Forthal

James Foulds

Tanja Franciskovic

Sophia Frangou

Emma Fransson

Mark Freestone

Paul French

Thomas Frodl

Leiwen $\mathrm{Fu}$

Romayne Gadelrab

Peter Gallagher

Richard Gallagher

Satheesh Kumar Gangadharan

Caroline Gao

Grace Gao

Maria Gardani

Elena Garralda

Chiara Gastaldon

Graham Gee

Manoj George

Galit Geulayov

Nassir Ghaemi

Cassandra Gheorghe

Abhishek Ghosh

Domenico Giacco

Kristina Gicas

Lucas Giner

Michael Gitlin

Paul Glue

Brandon Goldstein

Clara González Sanguino

Sarah Gordon

Christopher Gordon

Louise Gorman

Philippos Gourzis

Linda Grabbe
Denford Gudyanga

Amelia Gulliver

Mayank Gupta

Kassahun Habtamu

John Haltigan

Johannes Hamann

Elliot Hampsey

Jens Peter Hansen

Ketil Hanssen-Bauer

Helen Harding

Stephanie Hare

Zinta Harrington

Nigel Harvey

Alex Haslam

Tanya Hauck

Aaron Hauptman

Philip Hazell

Claire Henderson

Urs Hepp

Catherine M. Herba

Holly Herberman Mash

Helen Herrman

Morten Hesse

Walter Hewer

Madelyn Hsiao-Rei Hicks

Christoph Hiemke

Donald Hilty

Lindsey Hines

Yoshiyuki Hirano

Anders Hjern

Roger Ho

Jennifer Hoblyn

Nico Hoertel

Maurício Hoffmann

Josanne Holloway

Frank Holloway

Philipp Homan

Ismail Hosen

Sahadat Hossain

Louise Howard

Yong-Dong $\mathrm{Hu}$

Yueqin Huang

Dieneke Hubbeling

Peter Hughes

Clara Humpston

Richard Hunter

Muhammad Husain

Nusrat Husain

Mark Huthwaite

Christoph Hörmann

Eduardo Iacoponi

Maree Inder

Felice Jacka

Brian Jacobs

Nicholas Jacobson

Sneha Jadhav

Peter James

Imke Jansen

Marek Jarema

Arash Javanbakht

Manish Jha

Sheri Johnson

David Jolley

Edgar Jones

Brett Jones
Mark Jordans

Martin Jorgensen

Marin Jukic

Mario Juruena

Thomas Kabir

Michael Kaess

Ashraf Kagee

Richard Kanaan

Stephanie Kandsperger

Hitoshi Kaneko

Tyler Kaster

Cornelius Katona

Tamar Katz

Kenneth Kaufman

Harneet Kaur

Matthew Kemptom

Michael Kerr

Jess Kerr-Gaffney

Helen Killaspy

Irina Kinchin

Patricia Kinser

Tayyeba Kiran

James Kirkbride

Steve Kisely

Taro Kishi

Stefan Kloiber

Günter Klug

Anke Kobach

Yasin Koc

Roman A Koposov

Giorgio Kotzalidis

Golo Kronenberg

Krzysztof Krysta

Ashok Kumar-Jainer

Veena Kumari

Andre Kuntz

Hiroshi Kunugi

Wiliiam LaFrance

Danielle Lamb

Matthew Large

Celine Larkin

Rachel Latham

Sharon Lawn

Stephen Lawrie

Edouard Leaune

Daniel Leightley

Belinda Lennox

Eric Lenze

Peter Lepping

Glyn Lewis

Roberto Lewis-Fernandez

Zezhi Li

Ming Li

Rodrigo Lima

Jennie Lister

Kathy Liu

Xufeng Liu

Shan-Yu Liu

Adrian Lloyd

Carmen Lopez

Pablo Lopez

Bernd Lowe

Bartosz Łoza

Peter Lucassen

Mario Luciano 
Cecilia Lundin

Tom Lundin

Angela Lupattelli

Andrew Lustig

Jurjen Luykx

Sean Lynch

Zaza Lyons

Deirdre MacManus

Mariana Maciel

Ambra Macis

Nicola Magnavita

Kathryn Magruder

Rohan Mahabaleshwarkar

Rajneesh Mahajan

Jennifer Manuel

Chen Mao

Suzanne Mares

Joanna Martin

Denise Martin

Allison Marziliano

Joanna Maselko

Jolanta Masiak

Lorenzo Mazzarini

Rosalind McAlpine

Jane McClinchy

David McDaid

Lauren McGillivray

Andrew McKechanie

Sally McManus

Roisin McNaney

Fiona Mcnicholas

Alan Meehan

Werkua Mekonnen

Habtamu Mekonnen

João Campos Mendes

Matthew Menear

Sarah Meshberg-Cohen

Ricarda Mewes

Christian Michael

Georgina Miguel Esponda

Ryan Mills

Supriya Misra

Francis Mitrou

Fhionna Moore

R. O. Moreira

Emma Morton

Christoph Mueller

Marco Mula

David Mumford

Trine Munk-Olsen

Esther Murray

Katherine Musliner

Matthias Müller

Dieter Naber

Jhunu Nahar

Miharu Nakanishi

Vamanjore Naushad

Laura Nawijn

David Ndetei

Diana Nechita

Katlyn Nemani

Minh-Hoang Nguyen

Neil Nixon

Evangelos Ntontis

Peter Nussbaum
Maria Nystazaki

David O' Regan

Aileen O'Brien

Joanne O'Connor

Rory O'Connor

Lydia O'Sullivan

Yuko Odagiri

Sherifat Oduola

Tadahisa Ohara

Tamara Ondruskova

Sian Oram

Abigail Ortiz

Mugtaba Osman

Edoardo Ostinelli

Dennis Ougrin

Simple Ouma

Clare Pain

Lawrence Palinkas

Fryni Panayidou

Daniel Pankowski

Carmine Pariante

Gordon Parker

Glenys Parry

Andrew Peckham

Julia Pei

Jonathan Peirce

Eva Petkova

Theodore Petti

Peter Phiri

Giulia Piazza

Alex Pieterse

Toby Pillinger

Vanessa Pinfold

Alexandra Pitman

Michael Pluess

Rob Poole

Richard Porter

Maj-Britt Rocio Posserud

Iskra Potgieter

Andrew Powell

Annabel Price

Stefan Priebe

Eleonora Prina

Soorej Jose Puthoopparambil

Yann Quide

Samah Rabei

Ravi Ramasamy

Rosalind Ramsay

Ambiga Ravi

Ursula Read

Sara Real Castelao

Cheryl Rees

Susan Rees

Sarah Reeve

Cheryl Regehr

Abraham Reichenberg

Lennart Reifels

David Reiss

Gaby Resmark

Charles Reynolds, III

Ali Rezaeisharif

Abdul Rhouma

Wagner Ribeiro

Simon Rice

Laura Ridgeway
Zoltan Rihmer

Louise Robinson

Jo Robinson

Tomos Robinson

Paul Robinson

Humbelina Robles Ortega

Deborah Robson

Liana Romaniuk

Stuart Rosen

Larissa Rossen

Ashok Roy

Paola Rucci

Antonio Ruiz Garcia

Augustus Rush

Torleif Ruud

Grace Ryan

Janus Rybakowski

Martha Sajatovic

Estela Salagre

Myrto Samara

Musa Sami

Rahil Sanatinia

María Dosil Santamaría

Vedat Sar

Amir Sariaslan

Alan Schatzberg

Diana Schendel

Stephen Schoenthaler

Jan Scott

Edward Selby

Lucas Sempe

Vaheshta Sethna

Nick Sevdalis

Arieh Shalev

Rohit Shankar

Jalil-Ahmad Sharif

Jenny Shaw

James Shearer

Bryony Sheaves

Bart Sheehan

Lin Shen

Andrew Shepherd

Sukhi Shergill

Mark Shevlin

Rahul Shidhaye

Sonali Shinde Tesia

Brian Shiner

Wendy Shoesmith

Gemma Sicouri

Derrick Silove

Benedetta Silva

Douglas Simkiss

Judit Simon

Alexander Simpson

Carra Simpson

Mark Sinyor

James Smith

Andrew Sommerlad

Anne Sonley

Faye Stanage

John Stancombe

Corbin Standley

Thomas Steare

Lucy Stephenson

Robert Stewart 
Anne Stiggelbout

Julia Stingl

John Stone

Rebecca Strawbridge

Sladjana Štrkalj Ivezić

Genichi Sugihara

Paul Summergrad

Lois Surgenor

Nursel Sürmelioğlu

Keith Syrett

Olalla Sáiz-Vazquez

Somayyeh Taklavi

Sandila Tanveer

Alvin Kuowei Tay

Neil Thomas

Lindsay Thomson

Gillian Thomson

Paul Tiffin

Ami Tint

Elena Toffol

Elena Tomba

Leonardo Tondo

Ivan Torres

Ellen Townsend

Derek Tracy

Samuel Tromans

Hector Tsang

Dimosthenis Tsapekos
Mekonnen Tsehay

Rebecca Turner

Bryan Tysinger

Michael Udedi

Rachel Upthegrove

Jolien van Breen

Rob van den Brink

Peter van der Velden

Patrizia Velotti

Pieter Ventevogel

Lucia Verginer

Simone Vigod

Birgit Vollm

Louiza Voniati

Robert Walker

Sonya Wallbank

Malin Wallin

Angel Wang

Wayne Warburton

Nicola Warren

Danuta Wasserman

Lauren Waterman

Marielle Wathelet

Rebecca Webb

Myrna Weissman

Karen Wetherall

Daniel Whiting

Richard Whittington
Alina Wilkowska

Jill Williams

Richard Williams

Chris Williams

Claire Wilson

Ceri Wilson

Robert Winterhalder

Michael Wise

Ben Hoi-Ching Wong

Amanda Woodrow

Adam Włodarczyk

Xiaoxian Xie

Min Yang

Xinhua Yang

Lakshmi N. Yatham

Yung-Chieh Yen

Dong Keon Yon

Roseline Yong

Allan Young

$\mathrm{Yu} \mathrm{Yu}$

Sedigheh Zabihi

Zainab Zadeh

Roland Zahn

Caroline Zangani

Daniel Zheng

Chuanjun Zhuo

Hannah Ziobrowski 